\title{
The Effect of Caloric Restriction and Treadmill Exercise on Reserpine-Induced Catalepsy in a Rat Model of Parkinson's Disease
}

\author{
Mohammad Reza Yazdian', Azam Khalaj ${ }^{2 *}$, Naser Kalhor ${ }^{3}$ \\ ${ }^{1}$ Department of Physiology, Faculty of Medicine, Qom Branch, Islamic Azad University, Qom, Iran \\ ${ }^{2}$ Department of Physiology, Faculty of Basic Sciences, Qom Branch, Islamic Azad University, Qom, Iran \\ ${ }^{3}$ Research Center of Stem Cells, Academic Center for Education, Culture and Research (ACECR), Qom, Iran
}

\section{A BSTRACT}

Introduction: Parkinson's disease (PD), a degenerative disorder commonly affects the extrapyramidal system, is the second most common neurodegenerative disorder after Alzheimer's disease. It has been shown that caloric restriction increases life expectancy, decreases the incidence of several age-related diseases, and preserves youth activities in a large scale of laboratory animals. Several studies have revealed that exercise may have neuroprotective effects and increase neurogenesis and angiogenesis. Therefore, the aim of this study was to investigate the effect of caloric restriction diet on reserpine-induced catalepsy in a rat model of PD. Materials and Methods: 54 Wistar male rats were divided into 9 groups (control, exercise, PD, caloric restriction, PD + exercise, caloric restriction + exercise, $\mathrm{PD}+$ caloric restriction, caloric restriction $+\mathrm{PD}$ II, and PD + exercise +caloric restriction). PD was induced by administration of reserpine $(1 \mathrm{mg} / \mathrm{kg}$, i.p). The bar- test was used to measure cataleptic symptoms. All animals in the Exercise group were forced to run on a motorized treadmill (24 m/min for $20 \mathrm{~min}, 5$ days/week) for 3 weeks. Animals in the caloric restriction groups were under $30 \%$ caloric restriction diets. Results: Catalepsy was significantly lower in the caloric restriction + PD and PD + exercise groups than in the PD group. Furthermore, there was a significant decrease in catalepsy was observed in the PD + exercise + caloric restriction group compared to the PD. Conclusion: Caloric restriction of 30 percent and treadmill exercise can improve reserpine-induced catalepsy in an animal model of PD. In addition, application of caloric restriction before induction of PD may reduce the severity of complications in PD, including catalepsy.

\section{Key words:}

1. Caloric Restriction

2. Catalepsy

3. Exercise

4. Parkinson Disease

5. Rats

*Corresponding Author: Azam Khalaj

E-mail:A_khalaj@hotmail.com 


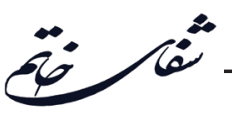

\title{
اثر محدوديت كالرى و ورزش تردميل بر كاتاليسى ناشى از رزريين در مدل موش صحرايى بيمارى

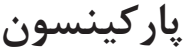

\author{
محمدرضا يزديان'، اعظم خلج"ّ، ناصر كلهرّ" \\ 'كروه فيزيولوزى، دانشكده يزشكى، واحد قم، دانشكاه آزاد اسلامى، قم، ايران

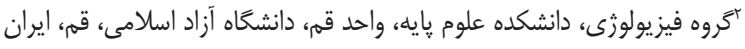 \\ "مركز تحقيقات سلولهاى بنيادى، جهاد دانشَاهى (ACECR) ، قم، ايران
}

اطلاعات مقاله:

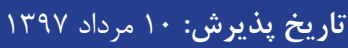

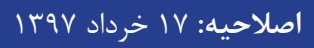

تاريخ دريافت: 9 اسفند و9سا

كليد وازمها:

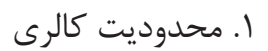

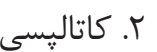

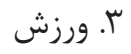

مقدمه: بيمارى پاركينسون يك اختلال تحليل برنده معمولاً بر دستخاه خارج هرمى اثر ميى گذارد كه بعد

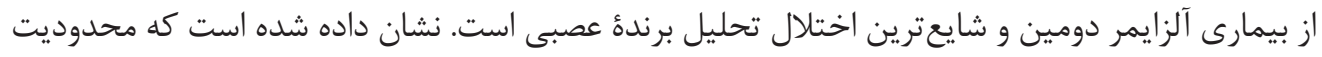

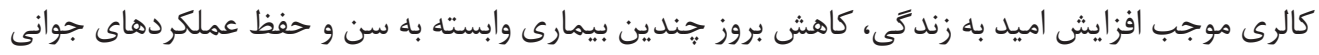

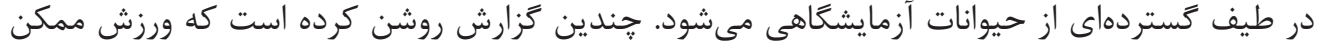

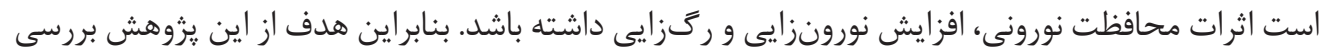

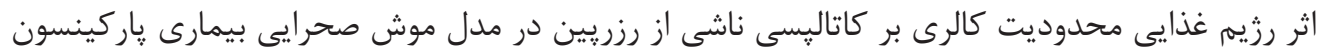

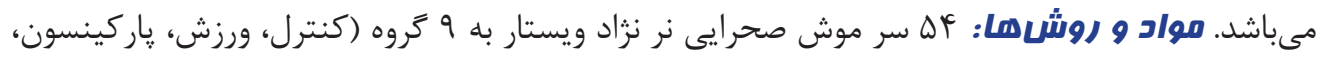

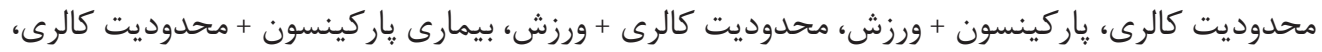

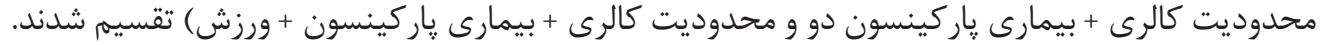

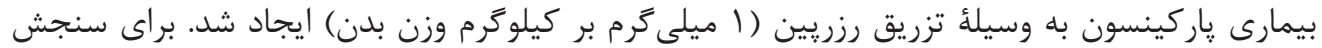

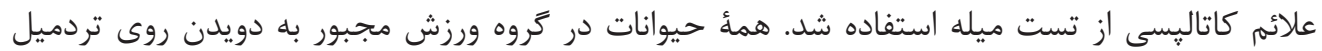

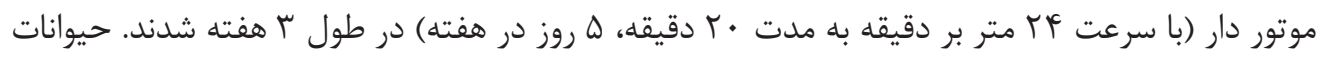

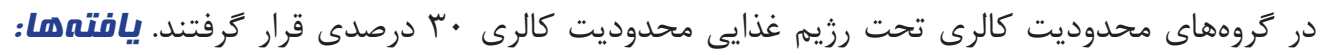

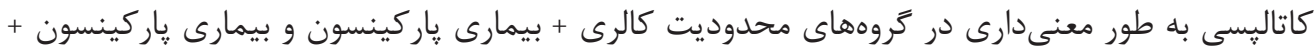

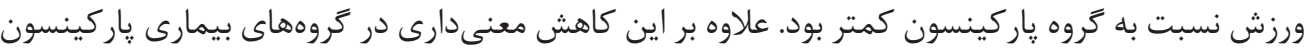

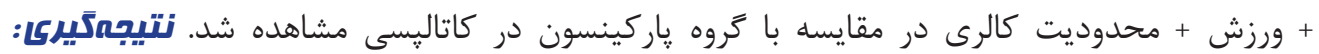

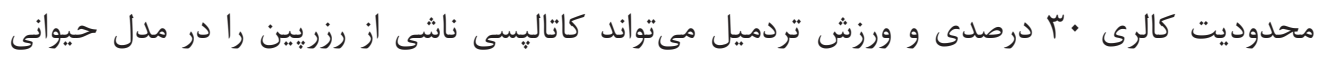

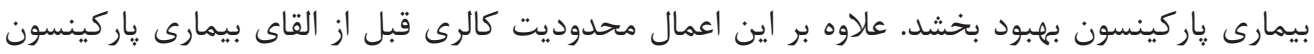

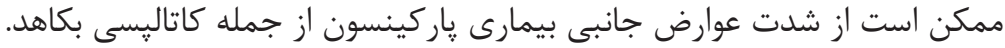

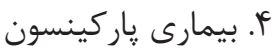

ه. له موش هاى صحرايى

" نويسنده مسئول: اعظم خلج

آدرس الكترونيكى: A_khalaj@hotmail.com 


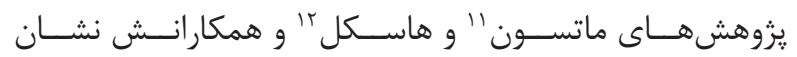

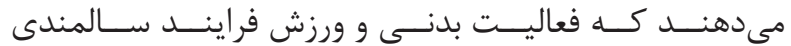

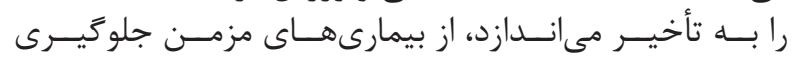

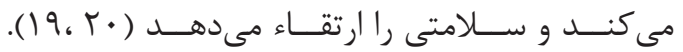

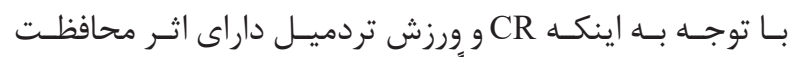

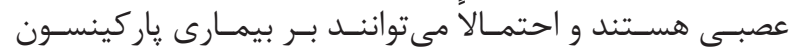

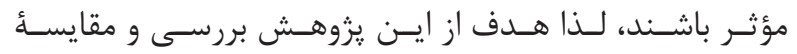

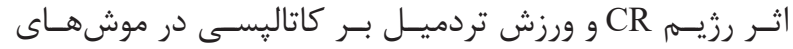

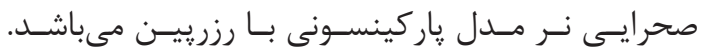

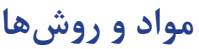
حيوانات مورد آزمايش

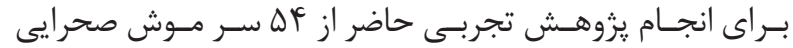

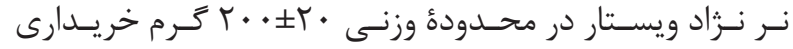

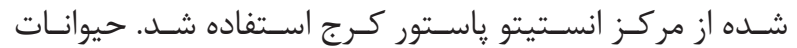

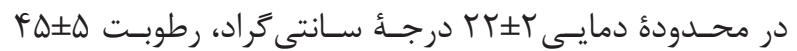

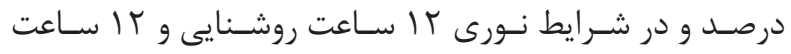

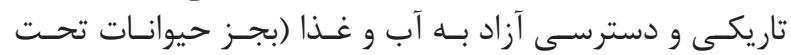

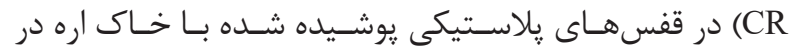

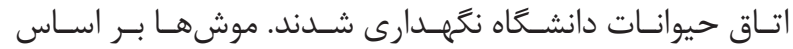

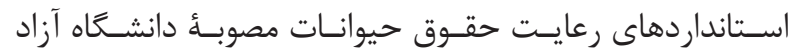

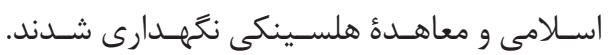

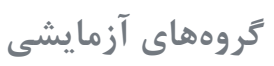

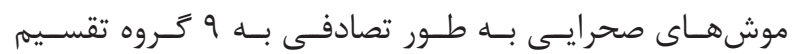

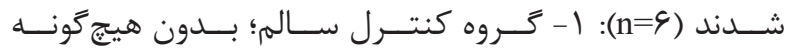

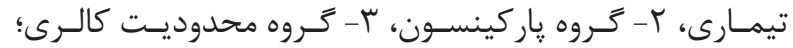

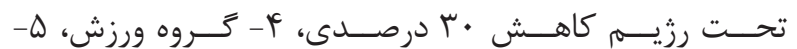

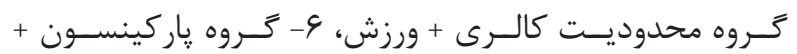

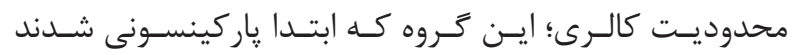

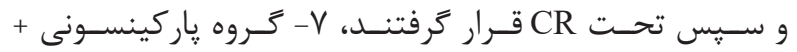

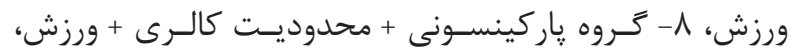

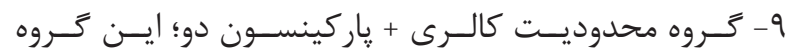

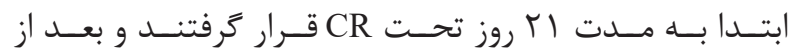

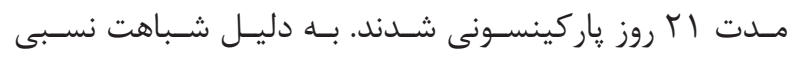

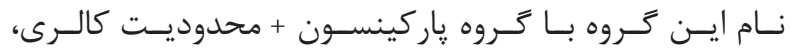

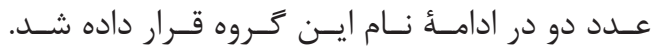

$$
\text { يروتكل اعمال محدوديت كالرى }
$$

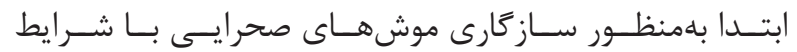

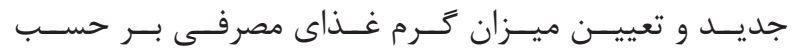

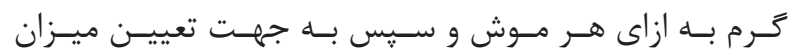

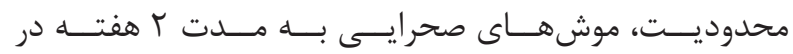

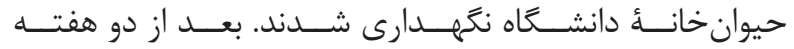

\section{${ }^{1}$ Substantia nigra}

${ }^{2}$ Basal ganglia

${ }^{3}$ Catalepsy

${ }^{4}$ Fixity of posture

${ }^{5}$ Caloric res triction

${ }^{6}$ Oxidative stress

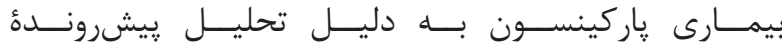

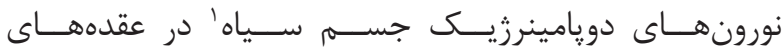

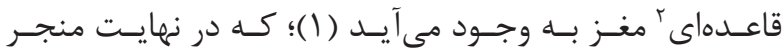

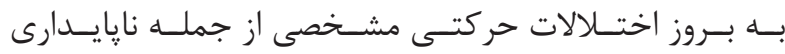

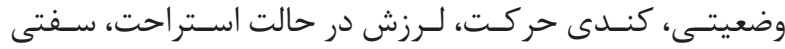

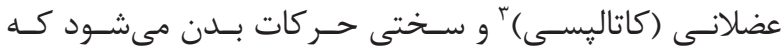

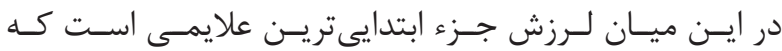

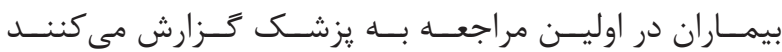

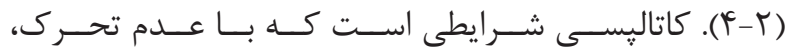

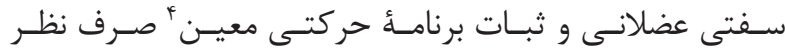

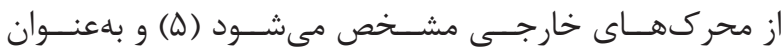

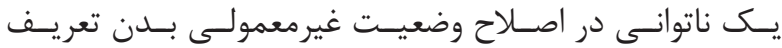

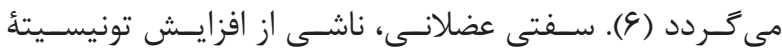

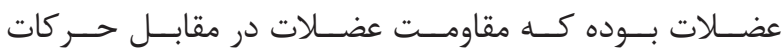

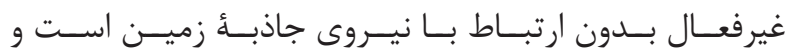

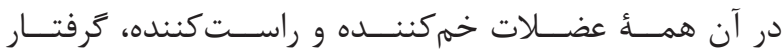

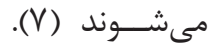

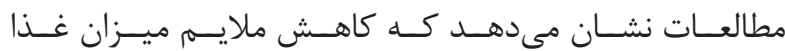

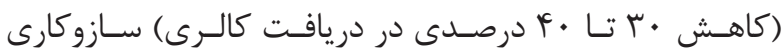

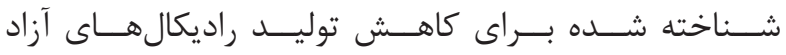

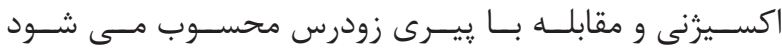

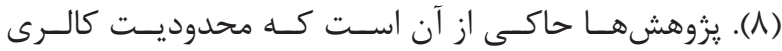
|كسئه

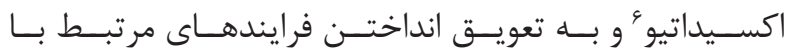

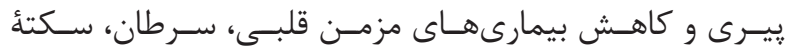

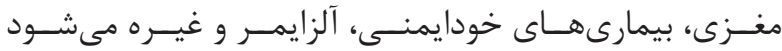

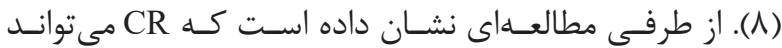

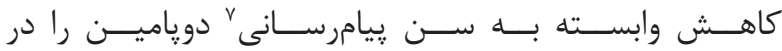

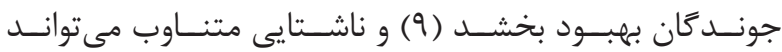

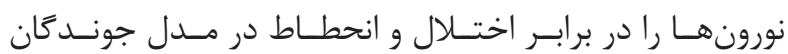

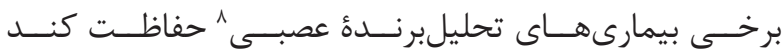

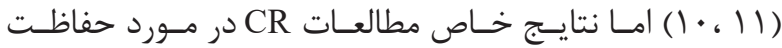

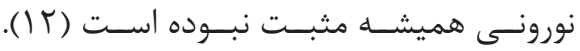

دو نـــوع ورزش وجـــــد دارد: ورزش اجبــارى و ورزش ارادى.

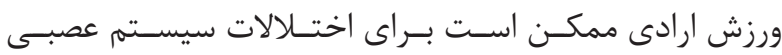

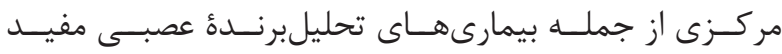

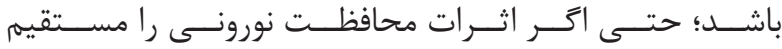

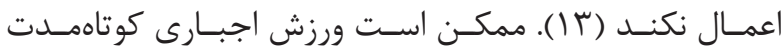

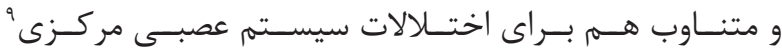
خــوب باشــ (CNS)

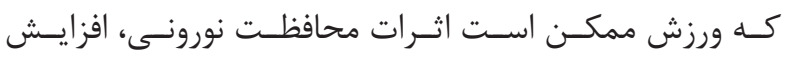

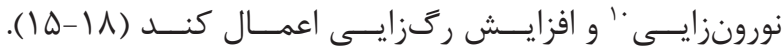

\footnotetext{
${ }^{7}$ Signaling

${ }^{8}$ Neurodegenerative

${ }^{9}$ Central nervous sys tem

${ }^{10}$ Neurogenesis

${ }^{11}$ Mattson

${ }^{12}$ Haskell
} 


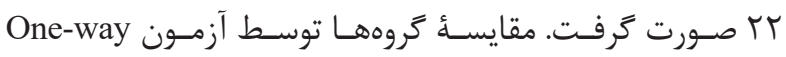
ANOVA

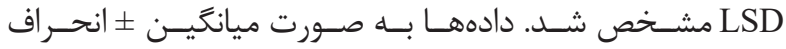

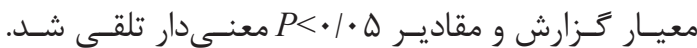

بافته ها

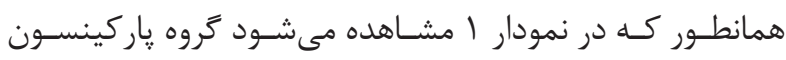

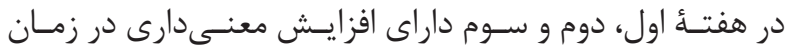

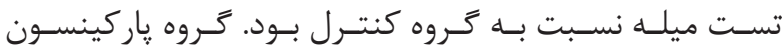

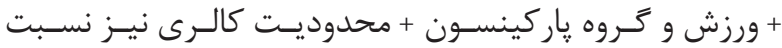

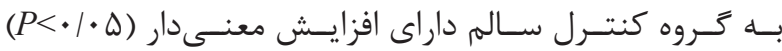

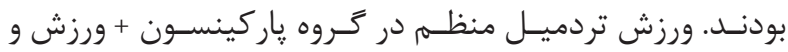

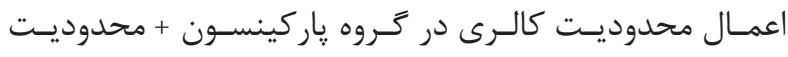

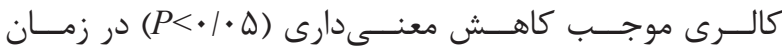

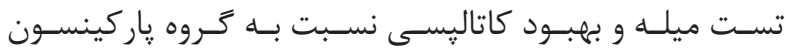

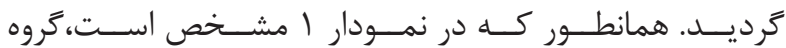

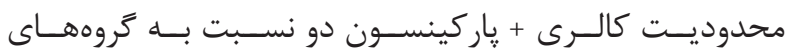

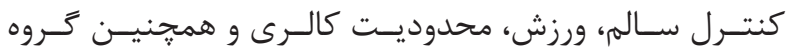

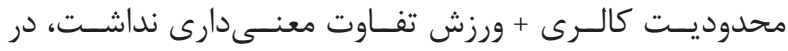

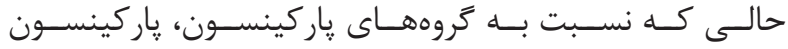

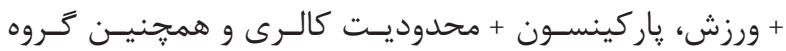

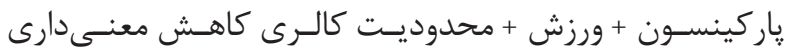

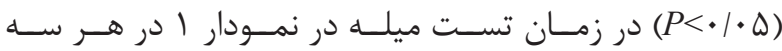

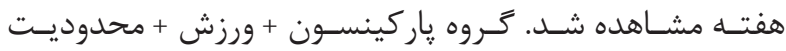

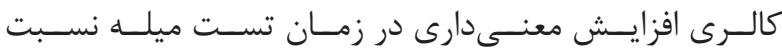

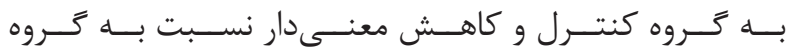

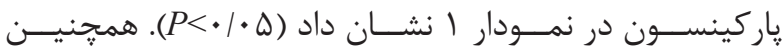

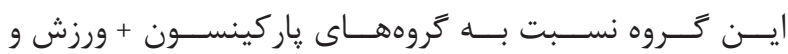

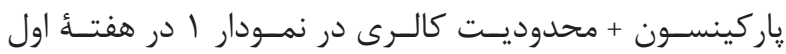

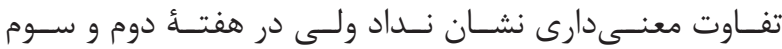

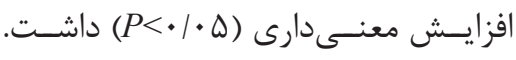

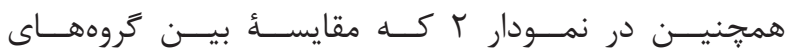

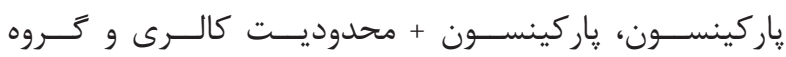

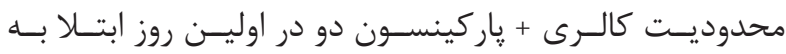

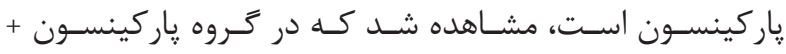

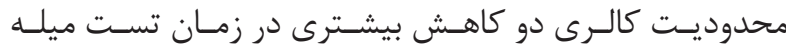

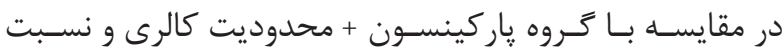

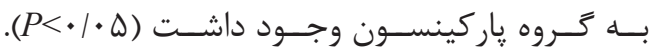

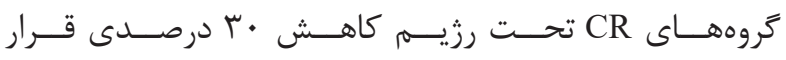

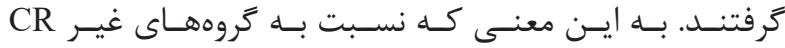

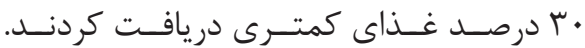

ابججاد بيمارى گيار كينسون

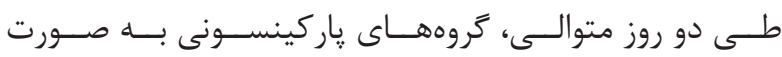

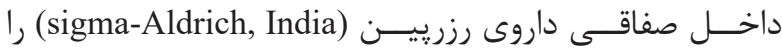

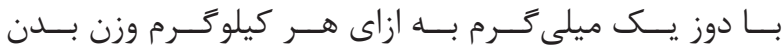

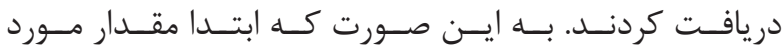

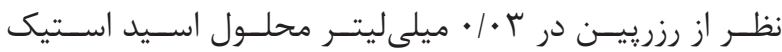

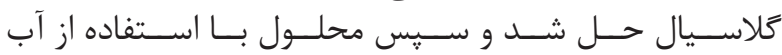

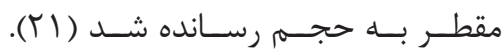

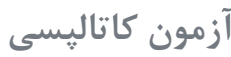

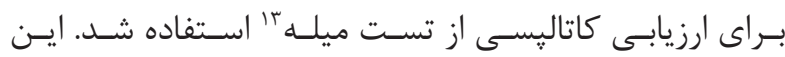

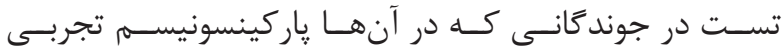

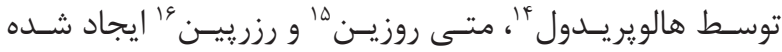

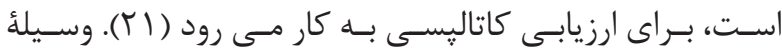

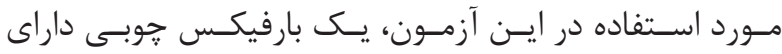

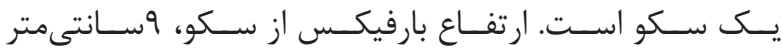

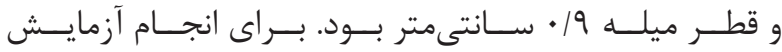

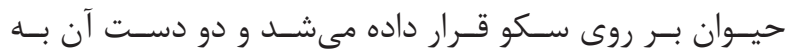

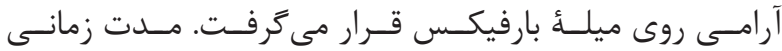

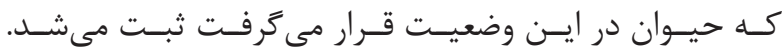

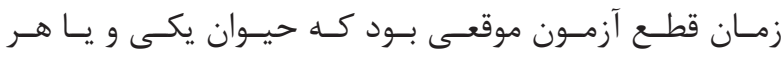

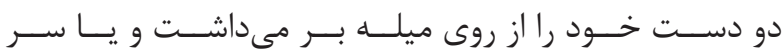

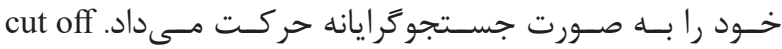

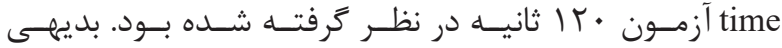

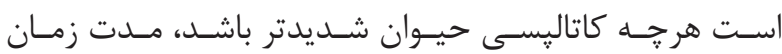

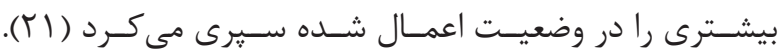

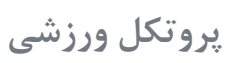

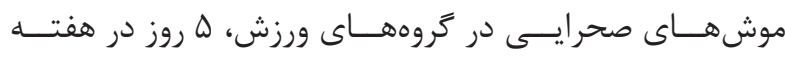

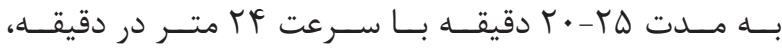

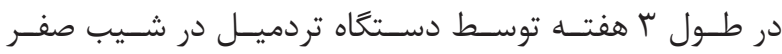

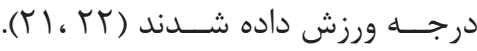
آناليز آمارى آناليـز دادههــاى حاصـل بــاســـفاده از نرمافـزار SPSS ويرايسـ

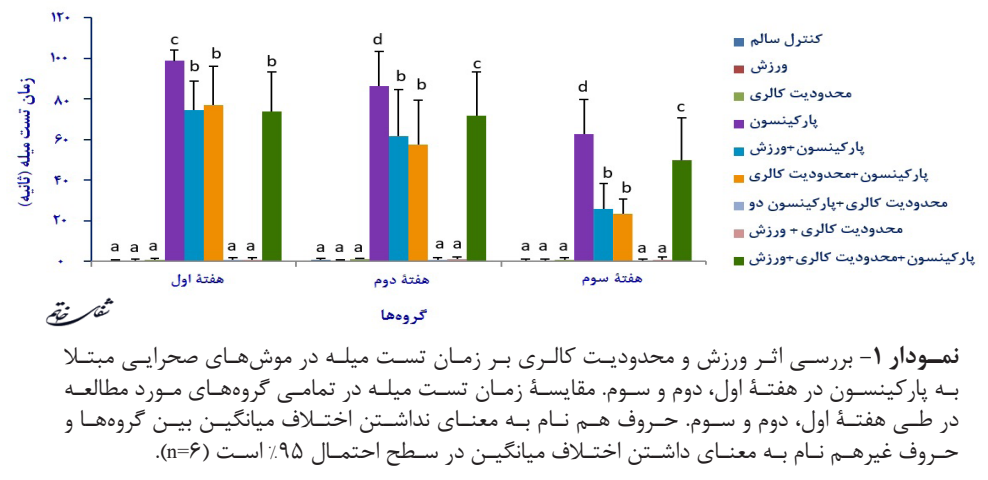

${ }^{13}$ Bar test

${ }^{14}$ Haloperidol
${ }^{15}$ Metyrosine

${ }^{16}$ Reserpine 


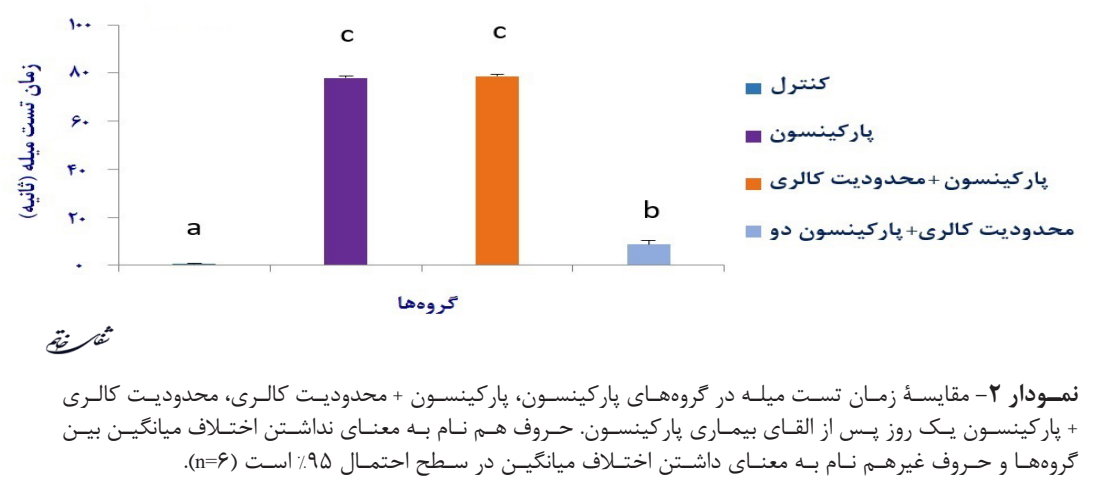

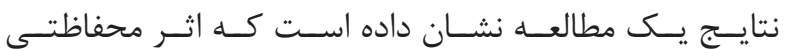

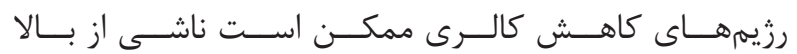

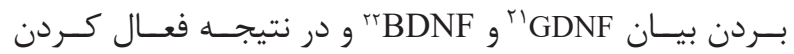

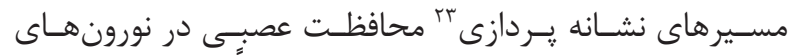

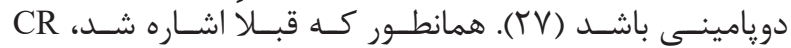

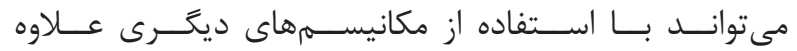

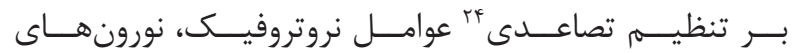

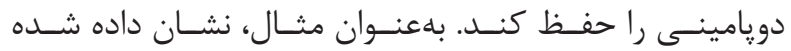

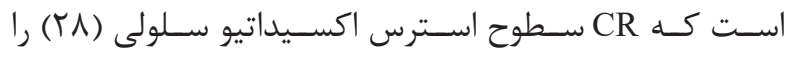

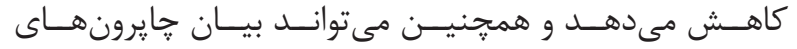

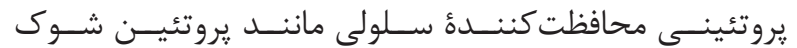

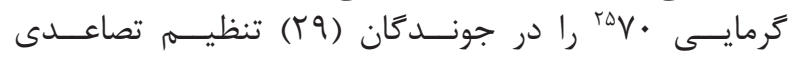

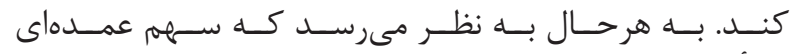

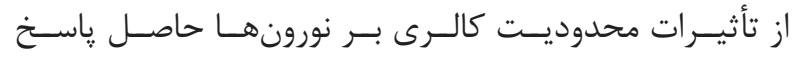

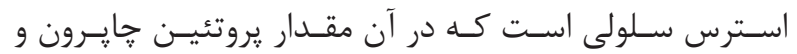

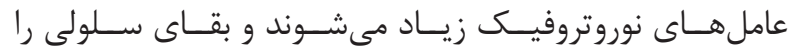

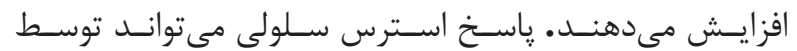

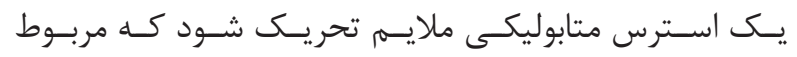

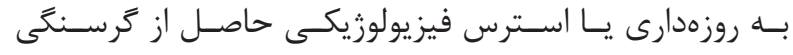

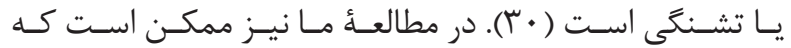

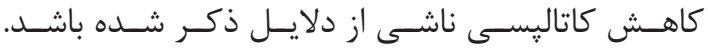

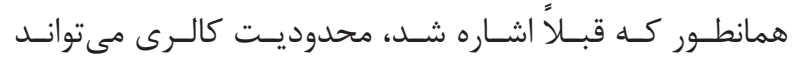

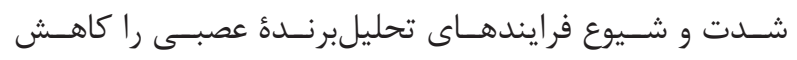

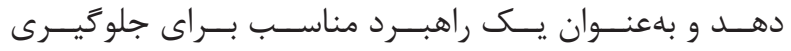

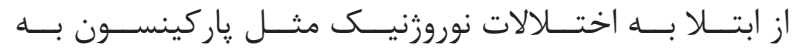

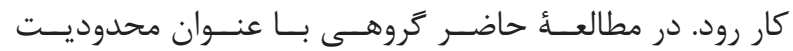

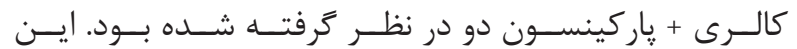

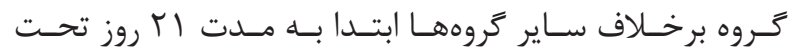

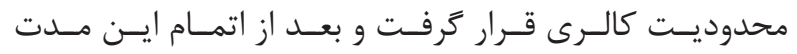

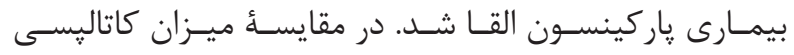

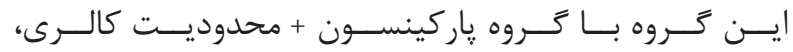

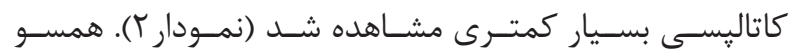

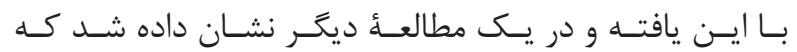

${ }^{17}$ Ad libitum

${ }^{18}$ Peroxisome proliferator-activated receptors

${ }^{19}$ Chaperone

${ }^{20}$ Neurotrophic factors

${ }^{21}$ Glial cell-derived neurotrophic factor
بحث و نتيجهَ

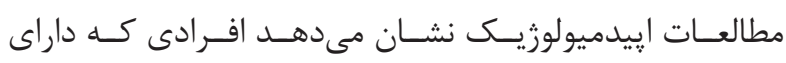

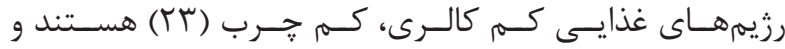

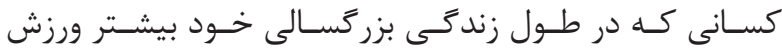

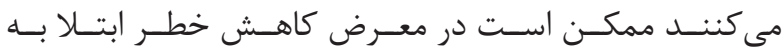

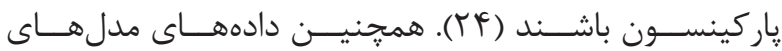

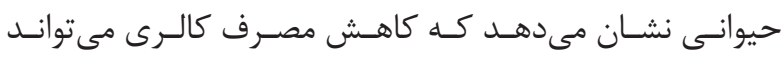

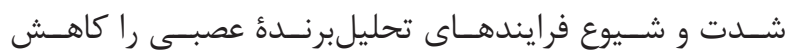

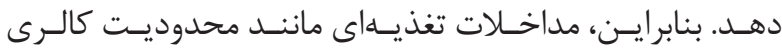

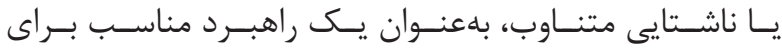

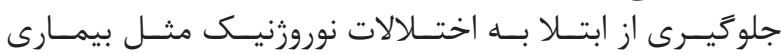

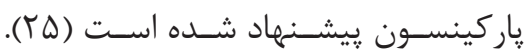

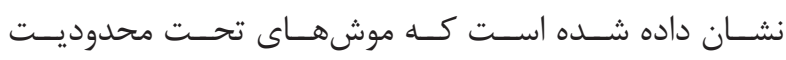

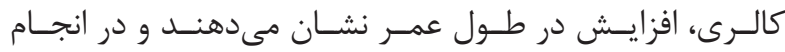

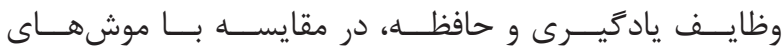

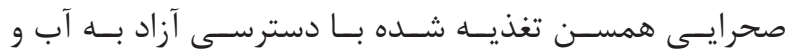

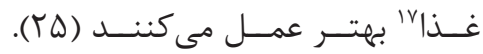

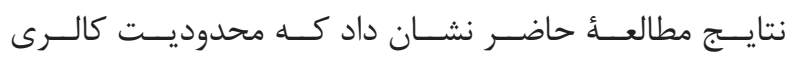

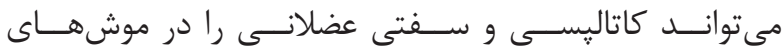

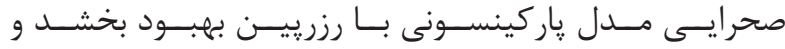

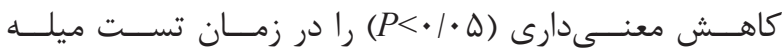

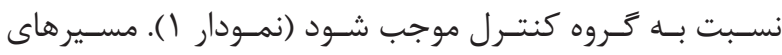

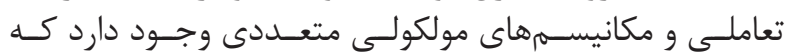

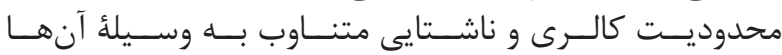

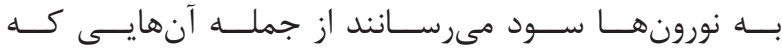

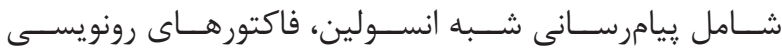
sirtuins ، FoxO

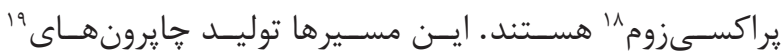

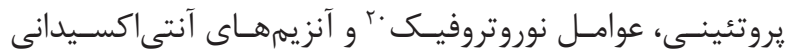

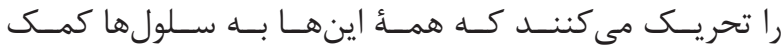

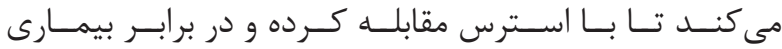

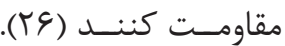

\footnotetext{
${ }^{22}$ Brain-derived neurotrophic factor

${ }^{23}$ Signal transduction

${ }^{24}$ Up-regulation

${ }^{25}$ Heat shock protein 70
} 
داده اسـت كــهـ ورزش موجــب بهبــود اختــلالات فيزيكـى و

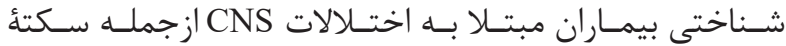

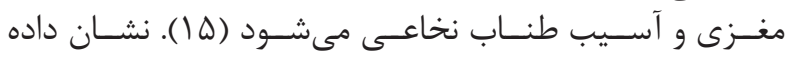

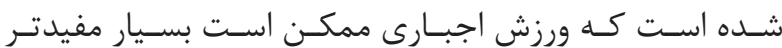
از ورزش اختيـارى باشــد (YF)

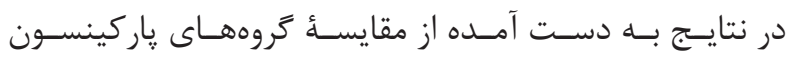

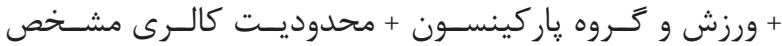

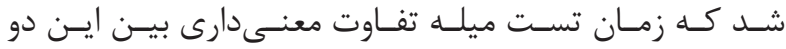

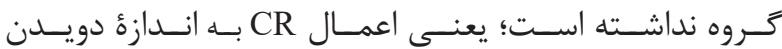

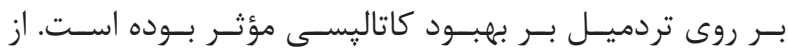

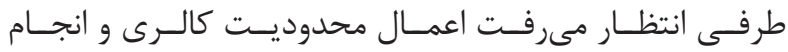

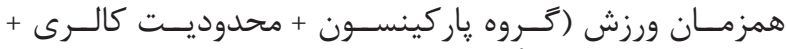

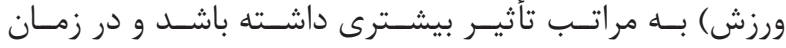

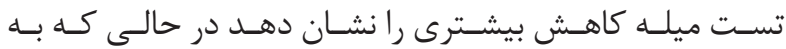

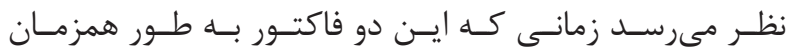

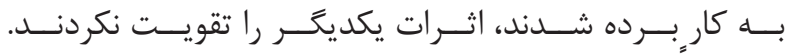

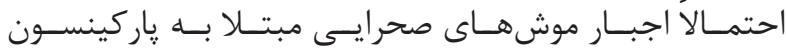

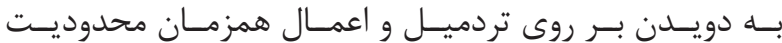

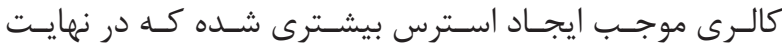

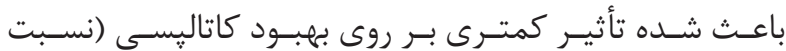

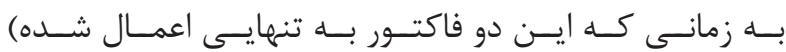

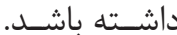

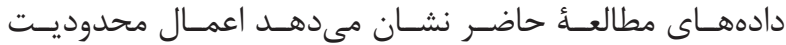

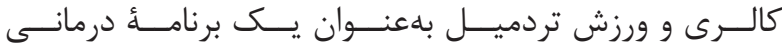

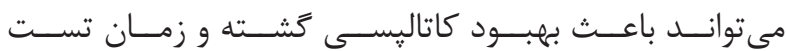

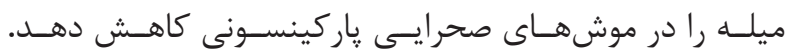

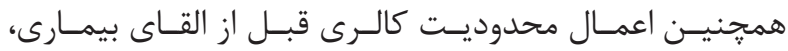

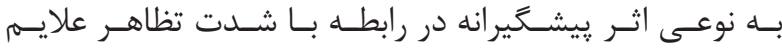

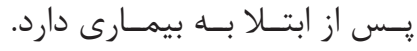

$$
\text { تشكر و قدردانى }
$$

از آقـاى دكتـر سـعيد حســينى و كليـهُ عزيزانـى كـهـ در انجـام

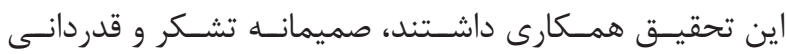

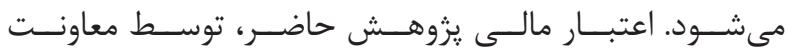

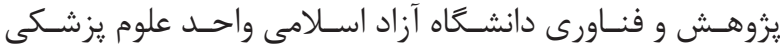

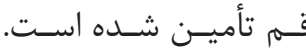

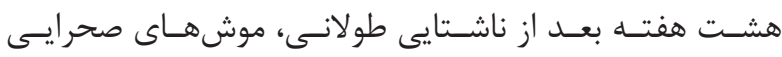

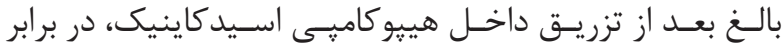

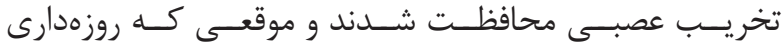

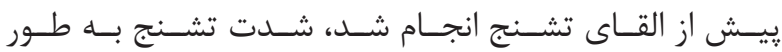

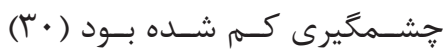

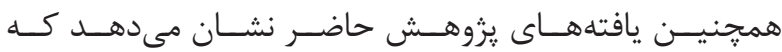

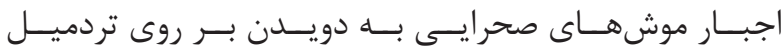

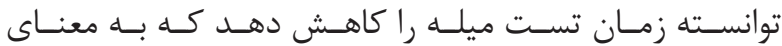

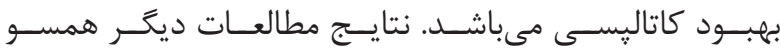

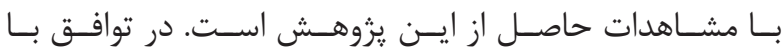

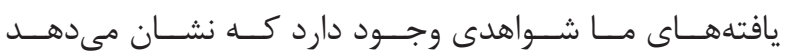

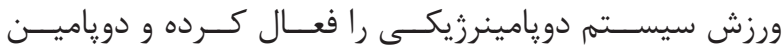

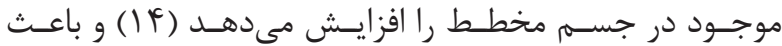

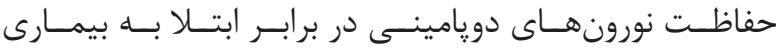

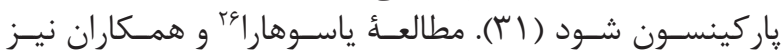

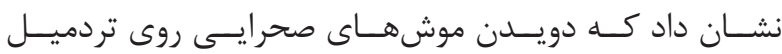

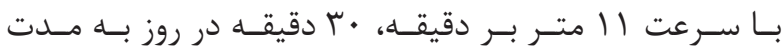

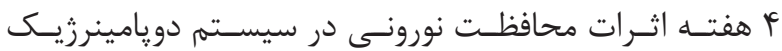

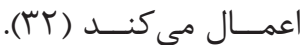

در مطالعـهلى كـهـ توســ احمــدى و همــكاران انجـام شــد،

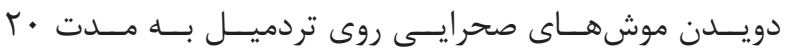

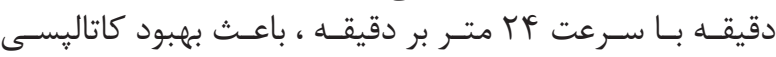

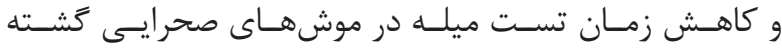
بـود (Y) به (Y).

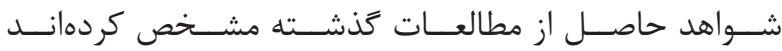

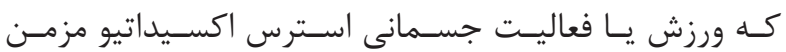

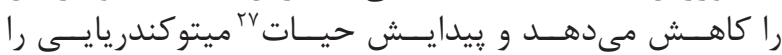

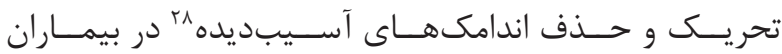

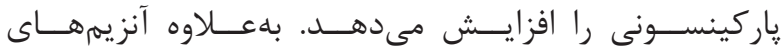

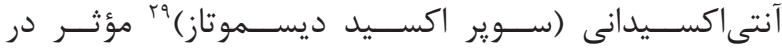

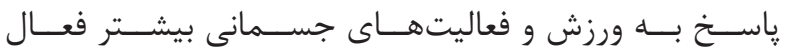

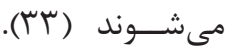

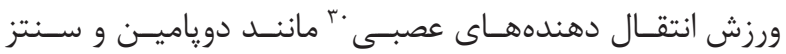

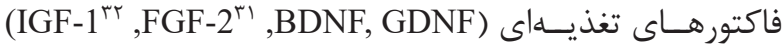

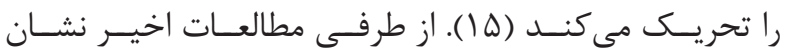

${ }^{26}$ Yasuhara

${ }^{27}$ Biogenesis

${ }^{28}$ Autophagy

${ }^{29}$ Superoxide dismutase (SOD)

\footnotetext{
${ }^{30}$ Neurotransmitters

${ }^{31}$ Fibroblast growth factor 2

${ }^{32}$ Insulin-like growth factor 1
} 
1. Chuang C-S, Su H-L, Cheng F-C, Hsu S-h, Chuang C-F, Liu C-S. Quantitative evaluation of motor function before and after engraftment of dopaminergic neurons in a rat model of Parkinson's disease. J Biomed Sci. 2010; 17(1): 9. doi: 10.1186/1423-0127-17-9.

2. Remy P, Doder M, Lees A, Turjanski N, Brooks D. Depression in Parkinson's disease: loss of dopamine and noradrenaline innervation in the limbic system. Brain. 2005 ; 128(6): 1314-22.

3. Starkstein SE, Merello M. Psychiatric and cognitive disorders in Parkinson's disease. J R Soc Med. 2003; 96(3): 151 .

4. Zimmermann R, Deuschl G, Hornig A, SchulteMönting J, Fuchs G, Lücking C. Tremors in Parkinson's disease: symptom analysis and rating. Clin Neuropharmacol. 1994; 17(4): 303-14.

5. Marrocco J, Mairesse J, Bucci D, Lionetto L, Battaglia G, Consolazione M, et al. Early life stress causes refractoriness to haloperidol-induced catalepsy. Mol Pharmacol. 2013; 84(2): 244-51.

6. Sanberg PR, Bunsey MD, Giordano M, Norman AB. The catalepsy test: its ups and downs. Behav Neurosci. 1988; 102(5(: 748-59.

7. Amidi M, Sarkaki A, Badavi M. Effect of intra striatum administration of MK-801 on muscular stifnes, straight lengthand electrical recording of globuspallidus in animal model of parkinson disease. PhD Thesis. Ahvaz Jondishapoor University of Medical Sciences, Medical Faculty. 2003.

8. Kouda K, Iki M. Beneficial effects of mild stress (hormetic effects): dietary restriction and health. J Physiol Anthropol. 2010; 29(4): 127-32.

9. Roth GS, Joseph JA. Cellular and molecular mechanisms of impaired dopaminergic function during aging. Annals of the New York Academy of Sciences. 1994; 719(1): 129-35.

10. Duan W, Zhang Z, Gash DM, Mattson MP. Participation of prostate apoptosis response-4 in degeneration of dopaminergic neurons in models of Parkinson's disease. Ann Neurol. 1999; 46(4): 587-97.

11. Duan W, Guo Z, Jiang H, Ware M, Li X-J, Mattson MP. Dietary restriction normalizes glucose metabolism and BDNF levels, slows disease progression, and increases survival in huntingtin mutant mice. Proc Natl Acad Sci U S A. 2003; 100(5): 2911-6.
منابع

12. Morgan WW, Richardson AG, Nelson JF. Dietary restriction does not protect the nigrostriatal dopaminergic pathway of older animals from low-dose MPTP-induced neurotoxicity. J Gerontol A Biol Sci Med Sci. 2003; 58(5): B394-B9.

13. O'dell S, Gross N, Fricks A, Casiano B, Nguyen T, Marshall J. Running wheel exercise enhances recovery from nigrostriatal dopamine injury without inducing neuroprotection. Neuroscience. 2007; 144(3): 1141-51.

14. Yoon M-C, Shin M-S, Kim T-S, Kim B-K, Ko I-G, Sung Y-H, et al. Treadmill exercise suppresses nigrostriatal dopaminergic neuronal loss in 6-hydroxydopamine-induced Parkinson's rats. Neurosci Lett. 2007; 423(1): 12-7.

15. Van Praag H, Christie BR, Sejnowski TJ, Gage FH. Running enhances neurogenesis, learning, and long-term potentiation in mice. Proc Natl Acad Sci U S A. 1999; 96(23): 13427-31.

16. Tillerson J, Caudle W, Reveron M, Miller G. Exercise induces behavioral recovery and attenuates neurochemical deficits in rodent models of Parkinson's disease. Neuroscience. 2003; 119(3): 899-911.

17. Kleim JA, Cooper NR, VandenBerg PM. Exercise induces angiogenesis but does not alter movement representations within rat motor cortex. Brain Res. 2002; 934(1): 1-6.

18. Swain RA, Harris AB, Wiener EC, Dutka MV, Morris HD, Theien BE, et al. Prolonged exercise induces angiogenesis and increases cerebral blood volume in primary motor cortex of the rat. Neuroscience. 2003; 117(4): 1037-46.

19. Haskell WL, Lee I-M, Pate RR, Powell KE, Blair SN, Franklin BA, et al. Physical activity and public health: updated recommendation for adults from the american college of sports medicine and the american heart association. Med Sci Sports Exerc. 2007; 39(8): 1423-34.

20. Mattson MP. Neuroprotective signaling and the aging brain: take away my food and let me run. Brain Res. 2000; 886(1): 47-53.

21. Khalaj A, Ahmadi R. The effect of treadmill exercise on catalepsy from reserpine-induced Parkinson model in diabetic male rat. Feyz Journal of Kashan University of Medical Sciences. 2016; 20(5): 397-404.

22. Murray DK, Sacheli MA, Eng JJ, Stoessl AJ. The effects of exercise on cognition in Parkinson's disease: a 


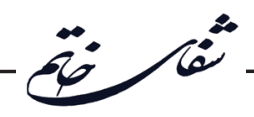

systematic review. Transl Neurodegener. 2014; 3(1): 5. doi: 10.1186/2047-9158-3-5.

23. Logroscino G, Marder K, Cote L, Tang MX, Shea S, Mayeux R. Dietary lipids and antioxidants in Parkinson's disease: a population-based, case-control study. Ann Neurol. 1996; 39(1): 89-94.

24. Sasco AJ, Paffenbarger RS, Gendre I, Wing AL. The role of physical exercise in the occurrence of Parkinson's disease. Arch Neurol. 1992; 49(4): 360-5.

25. Armentero M, Levandis G, Bramanti P, Nappi G, Blandini F. Dietary restriction does not prevent nigrostriatal degeneration in the 6-hydroxydopamine model of Parkinson's disease. Experimental Neurology. 2008; 212(2): 548-51.

26. Martin B, Mattson MP, Maudsley S. Caloric restriction and intermittent fasting: two potential diets for successful brain aging. Ageing Res Rev. 2006; 5(3): 332-53.

27. Maswood N, Young J, Tilmont E, Zhang Z, Gash DM, Gerhardt GA, et al. Caloric restriction increases neurotrophic factor levels and attenuates neurochemical and behavioral deficits in a primate model of Parkinson's disease. Proc Natl Acad Sci U S A. 2004; 101(52): 18171-6.

28. Barja G. Endogenous oxidative stress: relationship to aging, longevity and caloric restriction. Ageing Res
Rev. 2002; 1(3): 397-411.

29. Guo Z, Ersoz A, Butterfield DA, Mattson MP. Beneficial effects of dietary restriction on cerebral cortical synaptic terminals. J Neurochem. 2000; 75(1): 314-20.

30. Moghadami S, Mohammadzadeh E, Karimzadeh F. The effect of caloric restriction on the seizure behaviors in rats. Shefaye Khatam. 2013; 1(2): 21-6.

31. Howells FM, Russell VA, Mabandla MV, Kellaway LA. Stress reduces the neuroprotective effect of exercise in a rat model for Parkinson's disease. Behav Brain Res. 2005; 165(2): 210-20.

32. Tajiri N, Yasuhara T, Shingo T, Kondo A, Yuan W, Kadota T, et al. Exercise exerts neuroprotective effects on Parkinson's disease model of rats. Brain Res. 2010; 1310: 200-7.

33. Monteiro-Junior RS, Cevada T, Oliveira BR, Lattari E, Portugal EM, Carvalho A, et al. We need to move more: Neurobiological hypotheses of physical exercise as a treatment for Parkinson's disease. Med Hypotheses. 2015; 85(5): 537-41.

34. Kones R. Parkinson's disease: mitochondrial molecular pathology, inflammation, statins, and therapeutic neuroprotective nutrition. Nutr Clin Pract. 2010; 25(4): 371-89. 